\title{
Human Demodicosis: A Report of 5 Cases
}

\author{
Sharif Maraghi ${ }^{1,2,}$, Abdollah Rafiei $^{3}$, Gholam Abbas Kaydani ${ }^{4}$ \\ ${ }_{1}^{1}$ Department of Parasitology and Mycology, Arvand International University of Medical Sciences, Infectious and Tropical Diseases Research Center, Abadan, IR Iran \\ 2 Thalassemia and Haemoglobinopathy Research Center, Ahvaz Jundishapur University of Medical Sciences, Ahvaz, IR Iran \\ 3 Department of Parasitology, School of Medicine, Infectious and Tropical Diseases Research Center, Ahvaz Jundishapur University of Medical Sciences, Ahvaz, IR Iran \\ 4 Department of Laboratory Sciences, Paramedical School, Ahvaz Jundishapur University of Medical Sciences, Ahvaz, IR Iran \\ ${ }^{*}$ Corresponding author: Sharif Maraghi, Department of Parasitology and Mycology, Abadan Arvand International University of Medical Sciences, Infectious and Tropical Diseases \\ research Center, Thalassemia and Haemoglobinopathy Research Center, Ahvaz Jundishapur University of Medical Sciences, Ahvaz, IR Iran. Tel: +98-6113330678, Fax: +98-6112231325, \\ E-mail:maraghis@gmail.com. \\ Received: July 27, 2012; Revised: September 19, 2012; Accepted: September 30, 2012
}

\begin{abstract}
Introduction: Rosacea is a chronic skin disease affecting the facial area. The causative agents are unknown. Demodex mites can cause rosacea in human beings.

Case Presentation: In this article five cases of rosacea due to Demodex folliculorum are reported. The first case was a 26-year-old male wrestler. The second case was a 4-year-old-boy with immunodeficiency syndrome, the third case was a 29 -year-old lady, the fourth was a 7-year old boy and the fifth was a 36-year-old female. All patients were suffering from erythema, itching, inflammation and in some cases acne-like pustules in their face. The first case was suffering from lesions on his neck as well. All patients were referred to the Iran-Zamin diagnostic laboratory for fungal examination. Scraping from the lesions and slide preparation in $20 \% \mathrm{KOH}$ and microscopic examination revealed $D$. folliculorum. Scotch tape examination of the lesion on the neck of the first patient indicated the tinea versicolor. The patients referred to dermatologists for treatment.

Discussion: Clinical similarities of rosacea, fungal diseases and demodicosis might cause unsuitable therapy. Pretreatment examination and identification of disease agent lead to the appropriate management and treatment of the patients.
\end{abstract}

Keywords: Tinea versicolor; Rosacea

\section{Introduction}

Rosacea is a chronic disease affecting the skin of the nose, forehead and cheeks marked by flushing, followed by red coloration due to dilation of the capillaries with papules and acne like pustules. The causative agents and pathogenesis of rosacea are unknown and many factors such as genetics, infections, psychogenic factors and Demodex mites are contributing to this condition $(1,2)$. Demodex folliculorum and D. brevis are cosmopolitan (3).

The adult mites are only between $0.1 \mathrm{~mm}$ and $0.4 \mathrm{~mm}$ long, with $D$. brevis slightly shorter than $D$. folliculorum. They have a semi-transparent elongated body that consists of two fused segments. Eight short segmented legs attachedto the first body segment. Females are somewhat shorter and rounder than males. Both males and females have a genital opening, and fertilization is internal. Mating takes place in the follicle opening, and eggs are laid inside the hair follicles or sebaceous glands and the six- legged larvae hatch after 3- 4 days, andit takes about seven days for the larvae to develop into adults (4). Clinical manifestations of rosacea are varied. The infestation may be free of symptoms or the lesions might be as rose-red pustules and the most typical features of rosacea is characterized by the presence of an erythematous papule- pustule rash, mainly in the face and Inflammation in acute and chronic forms may occur $(5,6)$. Although $D$. folliculorum seems to be saprophyte and normal follicular inhabitant (7) but in many patients with rosacea- like lesions, Demodex mites were observed (8-11). Treatment options consist of several topical anti- parasitic and a few systemic drugs. Depending on the severity of the infection, topical application of metronidazole and oral use of antibiotics to prevent secondary bacterial infections is usually indicated $(12,13)$. In this article, five cases of $D$. folliculorum isolated from five patients with facial rosacea like referred to Iran- Zamin diagnostic laboratories for fungal examination in Ahvaz southwestern Iran are reported.

\section{Case Presentation}

\subsection{Case 1}

A-26-year-old wrestler man suffering from swelling and

Implication for health policy/practice/research/medical education:

The results of this study indicated that rosacea and acne- like demodicosis could be mis-diagnosed with dermatophytosis and pretreatment laboratory examination could lead the dermatologists to appropriate treatments.

Copyright (@) 2013, Ahvaz Jundishapur University of Medical Sciences; Licensee Kowsar Ltd. This is an Open Access article distributed under the terms of the Creative Commons Attribution License (http://creativecommons.org/licenses/by/3.0), which permits unrestricted use, distribution, and reproduction in any medium, provided the original work is properly cited. 
itching in the face, neck, trunk and upper arms for two years (Figure 1).

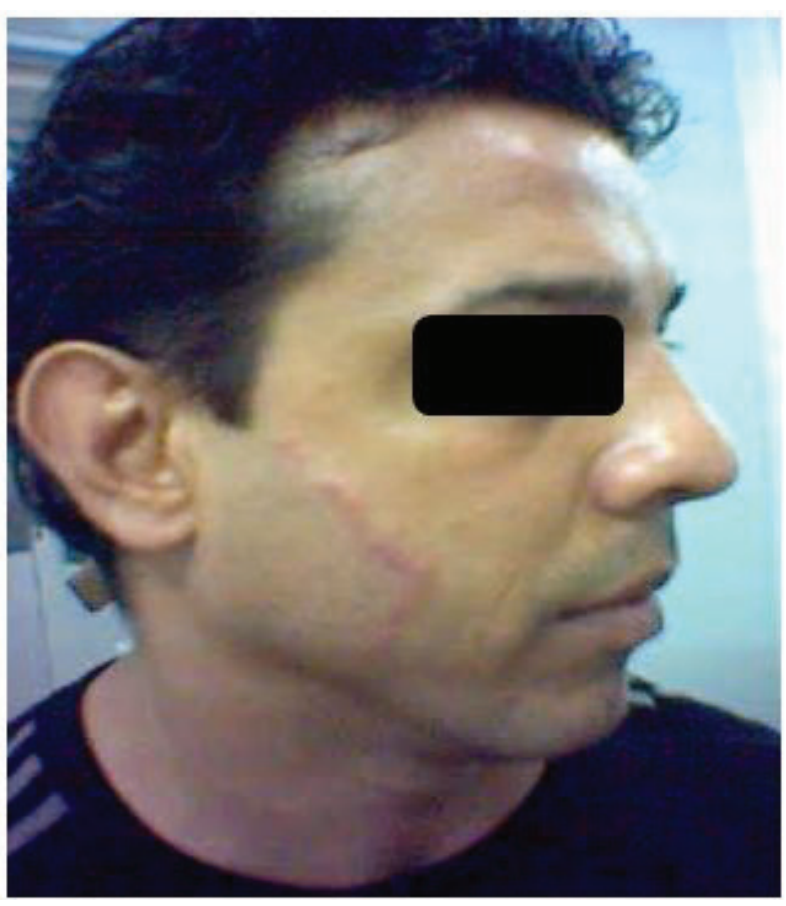

Figure 1. A- 26 Year Old Man Infected With D. folliculorum and Tinea versicolor

\subsection{Case 2}

A 4-year-old boy with immunodeficiency syndrome and facial lesion for four months (Figure 2)

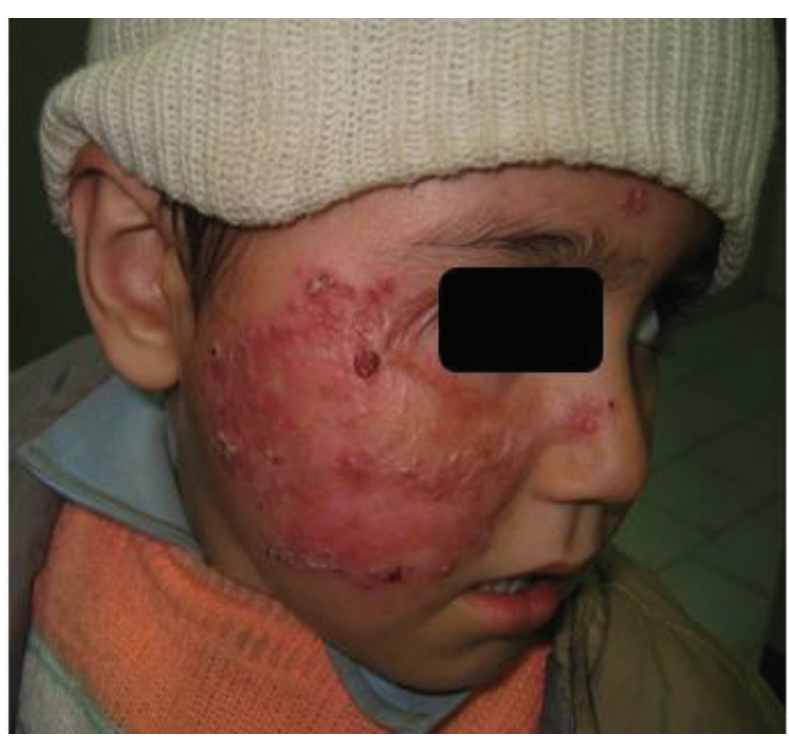

Figure 2. A- 4 Year Old Boy Infected With D. folliculorum

\subsection{Case 3}

A 29-year-old lady with acne- like lesion in her face for one year (Figure 3 ).

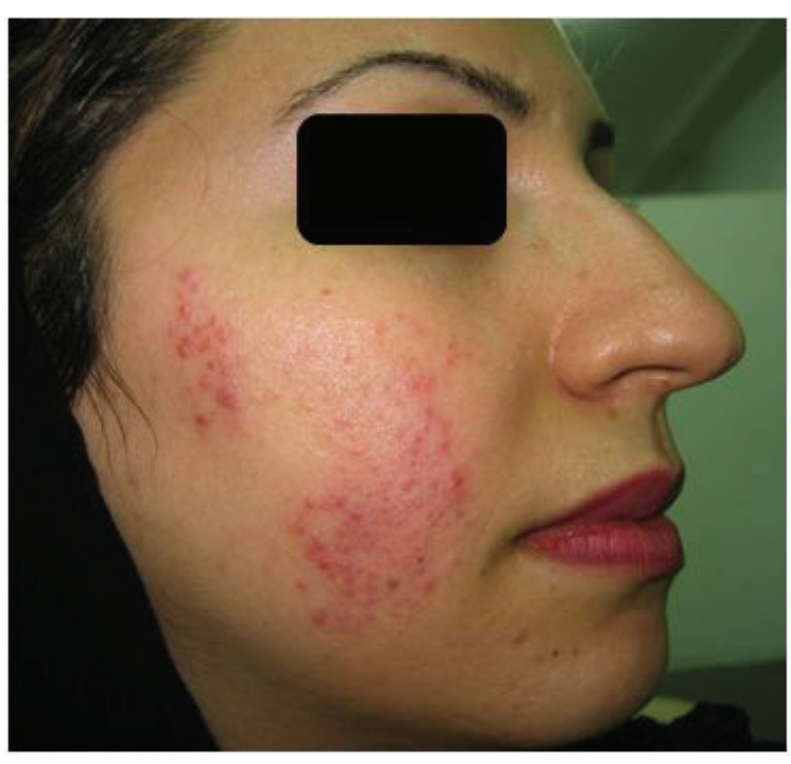

Figure 3. A 29 Year Old Lady Infected With D. folliculorum

\subsection{Case 4}

A 6-year-old boy with facial lesion for 6 months (Figure 4)

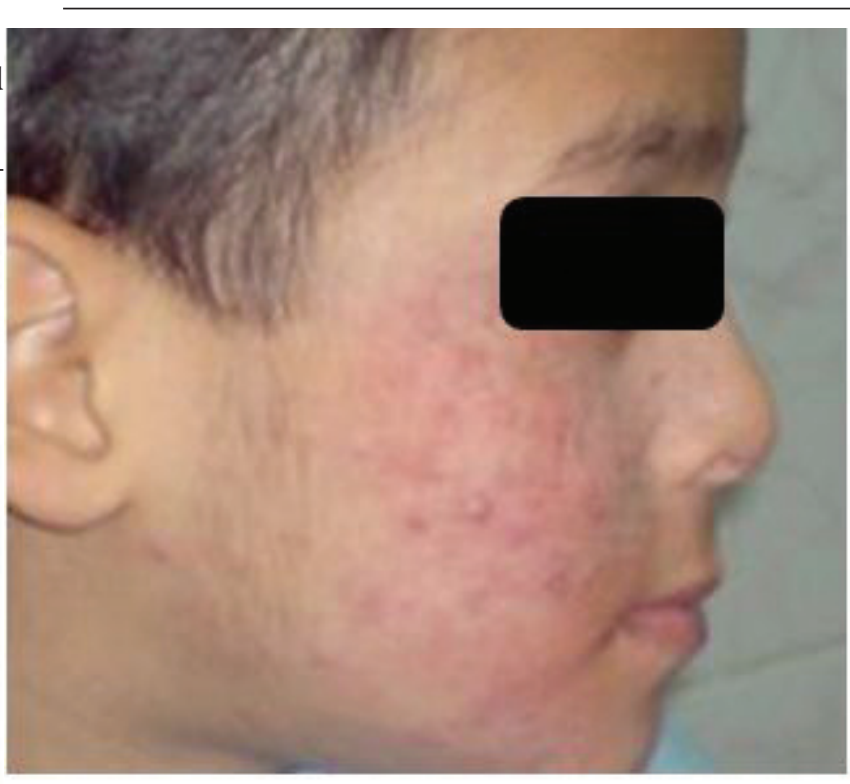

Figure 4. A 6 Year Old Boy Infected With D. folliculorum

The fifth case was not satisfying to take a picture from her lesion. All patients were prescribed with anti- histamine and anti- fungal medicine without any affecting during the period of infection. None of them had any di- 
agnostic examination prior treatment. The patients were referred to Iran-Zamin diagnostic laboratory for fungal examination. Scotch tape examination from the neck and trunk of the first patient revealed Tinea versicolor. Scraping from the facial lesions of all patients and smear preparation with $20 \% \mathrm{KOH}$ and microscopic examination indicated the $D$. folliculorum (Figure 5 ). All patients referred to dermatologists for treatment.

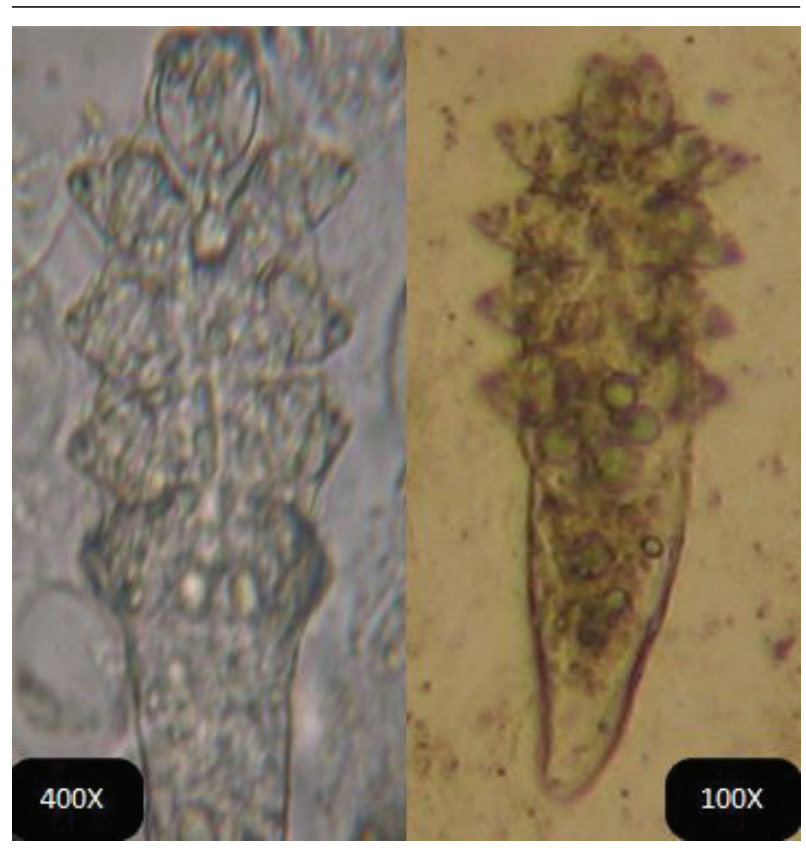

Figure 5. D. folliculorum mites isolated from patients referred to Iran-Zamin diagnostic laboratory

\section{Discussion}

The etiology of rosacea is not clear and many factors are involved in such chronic disorder (11). In this report, all patients were referred to the laboratory for fungal examination after administration of different medication without any success. Three of the subjects were male and two were female. The ages of the patients were 26, 4, 29, 7 and 36 year respectively. Only one of the subjects was infected with Tinea versicolor and the rest were negative for any fungal elements, while all of them were infested with $D$. folliculorum. The wrestler was present in the world wrestling championship and observed foreign wrestler with facial lesion.

Demodicosis is varied with age and highest rate of infestation is in the middle age and elderly (7) and seems to be sex dependent (10). Our cases were at the age of 26 , $4,29,7$ and 36 year respectively;three of them were male (60\%) and two (40\%) cases were female. Four patients were in healthy condition, but the second case with the age of 4 was immune-compromise. This symptom can increase the mite infestation $(14,15)$. Histopathologic studies of rosacea and non rosacea lesions indicated that $D$. folliculorum was significantly higher in rosacea patients (16). Treatment of rosacea with administration of corticosteroids did not cure the demodicosis and might cause $D$. burden (17), thus Demodex mite can cause rosacea or sever the lesion. The possibility of Demodex role in pathogenicity of skin lesion must be considered in laboratory examination of skin lesions, which can help the physicians for management the disease and administration suitable medication. Demodex mites can cause rosacea and acnelike lesions and in any facial lesion examination for dermatophytosis, demodicosis should be considered as well.

\section{Acknowledgements}

We would like to thank the cooperation of physicians and patients referred to Iran- Zamin diagnostic laboratory in Ahvaz southwestern Iran.

\section{Authors' Contribution}

None declared.

\section{Financial Disclosure}

No conflict of interest is declared by authors.

\section{Funding/Support}

None declared.

\section{References}

1. Del Rosso JQ. Update on rosacea pathogenesis and correlation with medical therapeutic agents. Cutis. 2006;78(2):97-100.

2. Goldsmith Lowell A. Fitzpatrick's Dermatology in General Medicine. 2012.

3. Woolley Tyler A. Acarology: mites and human welfare. 1988.

4. Rufli T, Mumcuoglu Y. The hair follicle mites Demodex folliculorum and Demodex brevis: biology and medical importance. A review. Dermatologica. 1981;162(1):1-11.

5. Pilehvar M, Zamanian A, Monsef AR, Mani-Kashani KH. Demodex folliculorum and rosacea. J Hamedan Univ Med Sci. 2001;22(4):5-8.

6. Sibenge S, Gawkrodger DJ. Rosacea: a study of clinical patterns, blood flow, and the role of Demodex folliculorum. JAm Acad Dermatol. 1992;26(4):590-3.

7. Pena GP, Andrade Filho JS. Is demodex really non-pathogenic? Rev Inst Med Trop Sao Paulo. 2000;42(3):171-3.

8. Bonnar E, Eustace P, Powell FC. The Demodex mite population in rosacea. JAm Acad Dermatol.1993;28(3):443-8.

9. Crawford GH, Pelle MT, James WD. Rosacea: I. Etiology, pathogenesis, and subtype classification. J Am Acad Dermatol. 2004;51(3):327-41.

10. Moravvej H, Dehghan-Mangabadi M, Abbasian MR, MeshkatRazavi G. Association of rosacea with demodicosis. Arch Iran Med. 2007;10(2):199-203.

11. Roihu Tia, Kariniemi Arja-Leena. Demodex mites in acne rosacea. J Cutan Pathol. 2006;25(10):550-552.

12. Junk AK, Lukacs A, Kampik A. [Topical administration of metronidazole gel as an effective therapy alternative in chronic Demodex blepharitis--a case report]. Klin Monbl Augenheilkd. 1998;213(1):48-50

13. Liu J, Sheha H, Tseng SC. Pathogenic role of Demodex mites in blepharitis. Curr Opin Allergy Clin Immunol. 2010;10(5):505-10.

14. Castanet J, Monpoux F, Mariani R, Ortonne JP, Lacour J Ph. Demodicidosis in an immunodeficient child. Pediatric dermatology. 2008;14(3):219-220.

15. Jansen T, Kastner U, Kreuter A, Altmeyer P. Rosacea-like demodici- 
dosis associated with acquired immunodeficiency syndrome. $\mathrm{Br}$ J Dermatol. 2001;144(1):139-42.

16. Monsef Alireza, Eghbalian Fatemeh. Histopathologic Study of Rosacea and the Role of Demodex Folliculorum. Iran J Pathol. 2006;(4):169-172.

17. el-Shazly AM, Ghaneum BM, Morsy TA, Aaty HE. The pathogenesis of Demodex folliculorum (hair follicular mites) in females with and without rosacea.J Egypt Soc Parasitol. 2001;31(3):867-75.

Please cite this paper as: Maraghi S, Rafiei A, Kaydani GA. Human

Demodicosis: A Report of 5 Cases. 2013; 6(5). DOI:10.5812/jjm.7465 